\title{
A SPECTROSCOPIC EVIDENCE FOR REACTIVE OXYGEN SPECIES PRODUCTION BY L-TRYPTOPHAN PHOTO OXIDATION FOR PGSA DENDRIMER-PROTOPORPHYRIN IX NANOFORMULATION
}

\author{
M. Suresh Kumar ${ }^{1,2, *}$, P. Aruna ${ }^{1}$ and S. Ganesan ${ }^{1}$ \\ ${ }^{1}$ Department of Medical Physics, Anna University, Chennai-600025, (TN) India. \\ ${ }^{2}$ Department of Physics, School of Basic Sciences, Vels Institute of Science, Technology \& \\ Advanced Studies, Pallavaram, Chennai-600117, (TN) India. \\ *Email: msknano@gmail.com
}

\begin{abstract}
Photodynamic therapy is an emerging modality for cancer treatment which involves the uptake of photosensitizer by cancer tissues followed by photo irradiation. Based upon the action of light activated photosensitizer in the excited state with the molecular oxygen, Reactive oxygen species (ROS) or free radicals formed due to electron transfer or energy transfer process are very toxic to cancer cells that can react with biomolecules and causes cell death. The present study describes the spectroscopic method of identification of ROS production for a model photosensitizer (PS), protoporphyrin IX (PpIX) in free and after loaded inside the biocompatible dendrimeric nano carrier with the help of decrease in tryptophan emission intensity at $286 \mathrm{~nm}$ excitation. The photochemical reaction of L-tryptophan with ROS after light treatment indicates, this may be due to photo-oxidation of tryptophan. This information supports to explore our study as spectroscopic evidence for monitoring the ROS production during photodynamic therapy.

Keywords: Photodynamic therapy, biocompatible anionic dendrimer, Reactive oxygen species, Protoporphyrin IX, Tryptophan, cancer, spectroscopy.
\end{abstract}

() RASĀYAN. All rights reserved

\section{INTRODUCTION}

Reactive Oxygen Species (ROS), plays a fascinating role in photodynamic therapy (PDT) related cell death with the combination of a photosensitizer (PS), a suitable light source that matches the maximum absorption peak of PS and molecular oxygen in the cancer tissue. Hydroxyl radials (OH), superoxide anion $\left(\mathrm{O}_{2}{ }^{-}\right)$, hydrogen peroxide $\left(\mathrm{H}_{2} \mathrm{O}_{2}\right)$ and singlet oxygen $\left({ }^{1} \mathrm{O}_{2}\right)$ are known as ROS which can be generated by Type I or Type II mechanism in PDT, regulates the cell death ${ }^{1,2}$. It is noteworthy that ROS are oxidizing agents, targets and directly react with many biological molecules of which amino acid residues (cysteine, methionine, tyrosine, histidine, and tryptophan) in proteins are of particular interest ${ }^{3,4}$. It is suitable to say, the production of ROS due to molecular reaction is dependent on PS concentration ${ }^{4}$. Juliana et al have described the possibility of ROS to react with different components of the cell in particular, tryptophan can be decayed by other ROS, but the ${ }^{1} \mathrm{O}_{2}$ can readily degrade tryptophan selectively by photo oxidation ${ }^{4,5}$ and also quantified the amount of singlet oxygen produced by a PS using a colorimetric assay ${ }^{4}$. A PDT agent with good ROS quantum yield is supposed to have good PDT efficacy. There is also a statement that, $\mathrm{H}_{2} \mathrm{O}_{2}$ one of the products of ROS is effective in producing DNA damage and lipid peroxidation, more appropriate than ${ }^{1} \mathrm{O}_{2}{ }^{2}$. But the ${ }^{1} \mathrm{O}_{2}$ generated information can be obtained from the oxidation of amino acid residues which are more liable to be attacked by ${ }^{1} \mathrm{O}_{2}{ }^{4,6}$ which is advantageous to quantify spectral fluorometrically ${ }^{7}$.In this context, native fluorescence spectroscopic study was carried out to explore whether it is possible to spot out the production of Reactive oxygen species (ROS) using chemical L-tryptophan for the photosensitizer (PpIX) and dendrimer G2.5-PpIX

Rasayan J. Chem., 12(3), 1027-1031(2019)

http://dx.doi.org/10.31788/RJC.2019.1235149

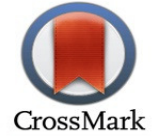


nano formulation after the light treatment. The photo oxidation of tryptophan which reflected in the decrease in the fluorescence emission spectra of tryptophan at $337 \mathrm{~nm}$ when excited at $286 \mathrm{~nm}$ draws the attention in our study to positively report this as spectroscopic evidence for the production of ROS in photodynamic therapy.

\section{Materials and Methods}

\section{EXPERIMENTAL}

Phloroglucinol and succinic acid were purchased from Sigma Aldrich, India to develop the biocompatible Phloroglucinol Succinic Acid (PGSA) dendrimer. The method used to develop each generation (G0.5G2.5) with an anionic surface charge for the PGSA dendrimer and the techniques used to confirm the presence of functional groups and their charges in the end product of every half and full generation terminals were already discussed by Murugesan et al ${ }^{9,14}$. Figure-1 shows the structure of anionic dendrimer of G2.5. The presence of PpIX inside the dendritic cavity of G2.5 has also been confirmed using UV-Vis., Fourier transforms Infer red (FTIR) spectroscopy techniques ${ }^{9}$, and Transmission electron microscopy $(\text { TEM })^{14}$.

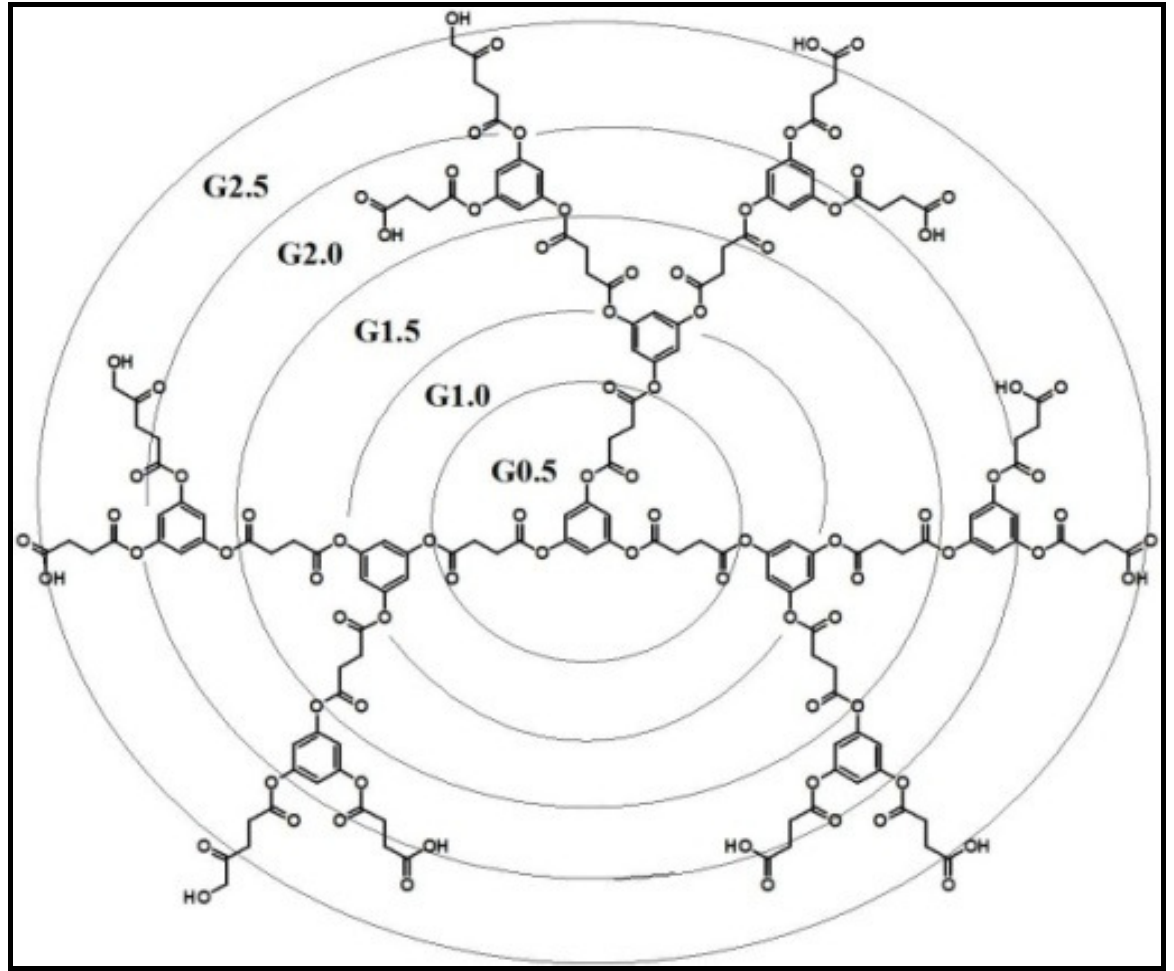

Fig.-1: Structure of Anionic Dendrimer of Generation 2.5

Photosensitizer (PpIX) and PpIX incorporated dendrimer of different concentrations (10, 25, 50, 75, 100 and $200 \mu \mathrm{g}$ ) were used for experiments for monitoring the photogeneration of singlet oxygen by assessing its reaction with tryptophan after light treatment with Xenon lamp, Red light $(630 \mathrm{~nm})$ of power $20 \mathrm{~mW}$ for 10 minutes. Various concentrations of PpIX and PpIX with dendrimer were incubated with $2 \mathrm{~mL}$ (100 $\mu \mathrm{M})$ of L-Tryptophan solution, $\mathrm{pH} 7.4$ in a 12 well plate for $1 \mathrm{~h}$. Following the light irradiation after the incubation period, tryptophan emission was measured with a fluorescence spectrophotometer (HitachiF4500, Tokyo, Japan) at $286 \mathrm{~nm}$ excitation.

\section{RESULTS AND DISCUSSION}

The steady-state fluorescence emission was recorded spectroscopically at $286 \mathrm{~nm}$ for $100 \mu \mathrm{M}$ concentration of L- tryptophan for which the emission peak was observed at $357 \mathrm{~nm}$ which is shown in Fig.- 2. PpIX in free and dendrimer encapsulated forms (G2.5-PpIX) in different concentrations (10, 25, $50,75,100$ and $200 \mu \mathrm{g}$ ) were subjected to light treatment for a period of 10 minutes after $1 \mathrm{~h}$ incubation with L-tryptophan $(100 \mu \mathrm{M})$ in dark. 
RASĀYAN J. Chem.

Vol. 12 | No. 3 |1027 - 1031| July - September | 2019

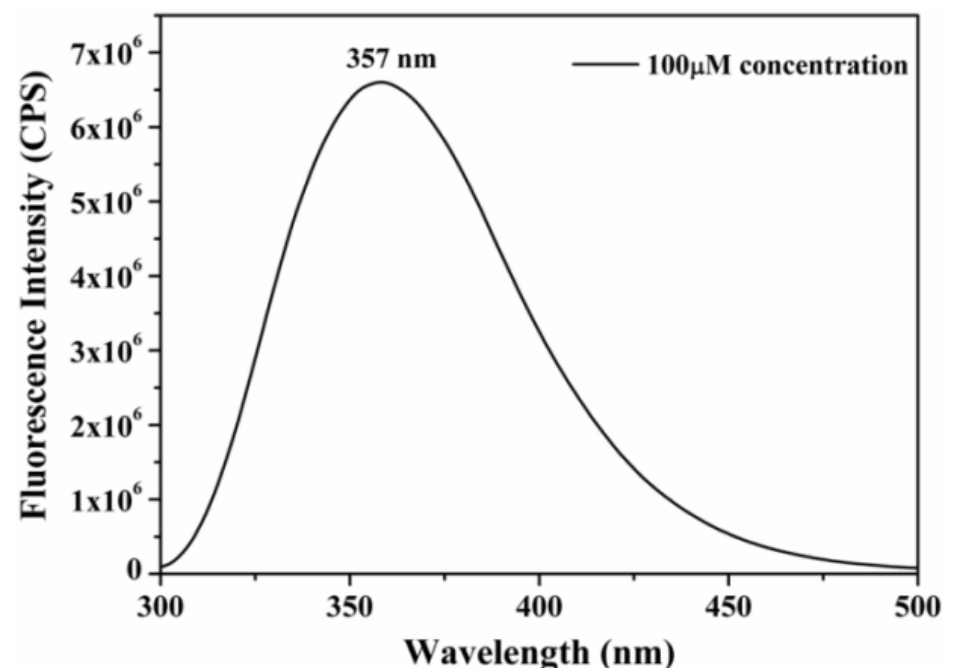

Fig.-2: Steady-state Fluorescence Emission Spectrum of Tryptophan @286 nm Excitation

After the light treatment, a blue shift $(337 \mathrm{~nm})$ in the emission spectrum of tryptophan was noticed which is about $20 \mathrm{~nm}$ from the raw tryptophan emission $(357 \mathrm{~nm})$ for both the PpIX in free and dendrimer encapsulated forms (G2.5-PpIX) at $286 \mathrm{~nm}$ excitation which is shown in Fig.-3a and 3b. Since, the excited photosensitizer plays a prominent role in self-quenching effect rather than oxidizing tryptophan molecules, this was also expected to encounter for PpIX in our case too when it is not entrapped inside the dendritic cavity.
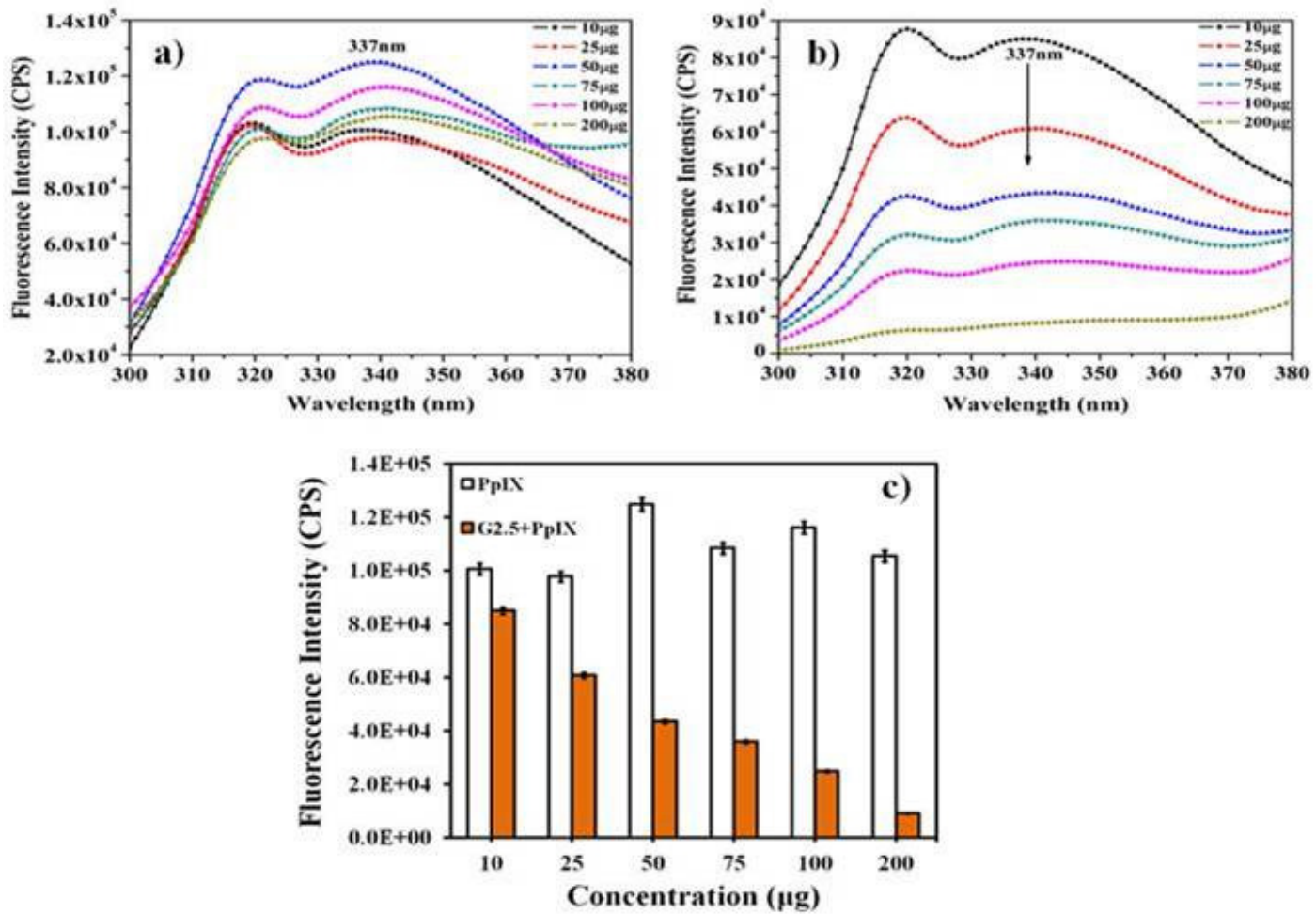

Fig.-3: Fluorescence Emission Spectra of Tryptophan @ 286nm Excitation for PpIX (a), G2.5+PpIX (b) and Maximum Fluorescence Emission Intensity of Tryptophan Vs Concentration of PpIX and G2.5+PpIX (c) after Light Treatment.

The speed of decrease in the fluorescence emission with $20 \mathrm{~nm}$ blue shift and absence of quenching effect (Fig.-3b) for the increase in concentration of G2.5-PpIX nano formulation distinctly provided information about the oxidation of tryptophan molecules which makes as clear to presume that, the decrease in the 
tryptophan emission can be related to ${ }^{1} \mathrm{O}_{2}$ production, which is predictable with the tryptophan as an in vitro study. This was not able to be identified for the free PpIX (Fig.-3a) due to its self-quenching behavior for an increase in concentrations after light treatment for the same time duration. Additionally, an emission peak at $320 \mathrm{~nm}$ was observed for all the treatments in our case (Fig.-3), which corresponds to the water Raman peak at $286 \mathrm{~nm}$ excitation ${ }^{8}$. It's reported that the water Raman peak moves from 311 $362 \mathrm{~nm}$ when the excitation wavelength changes from 280-320 nm ${ }^{10}$. In Fig.-3c, the maximum fluorescence emission intensity of tryptophan $(337 \mathrm{~nm})$ for free PpIX and G2.5-PpIX nano formulation after light exposure was comparatively shown in order to represent the occurrence of self quenching effect for PpIX even after 10 minutes of light exposure, due to this a gradual decrease in the tryptophan emission was not observed as concentrations were increased further. When compared to free PpIX, dendrimer (G2.5)-PpIX nano formulation expressed a gradual decrease in the tryptophan emission for an increase in concentration, in which the self-quenching effect was eliminated and explicitly confirms the photo oxidation of tryptophan when the free PpIX was loaded inside the dendritic cavity.

\section{CONCLUSION}

Reactive Oxygen Species in PDT which causes damage to the cells can interact with tryptophan present in cells and oxidize; which acts as a direct identification of ROS production after light treatment. So in order to understand this oxidation mechanism and to assess the ROS production, we have used chemical Ltryptophan and monitored the decrease in emission spectrum of tryptophan in the region between 330-340 $\mathrm{nm}(20 \mathrm{~nm}$ blue shift) by interacting with PpIX and dendrimer (G2.5)-PpIX formulation and subjecting to light treatment for a period of 10 minutes. From which we conclude that, PpIX incorporated inside the dendritic cavity expressed good photodynamic efficiency by oxidizing tryptophan than the free PpIX. As reported that direct spectroscopic evidence of singlet oxygen production in PDT is difficult to find ${ }^{2}$, due to its short life time within the cells ${ }^{11-13}$ and rapid reaction with biomolecules ${ }^{6}$, we have used this simple technique for the identification of ROS production with L-tryptophan; by means of just observing the photo oxidation of tryptophan spectroscopically with emission characteristics after PDT.

\section{ACKNOWLEDGMENT}

The work was supported by DAE-BRNS Grant (Ref. No. 2009/38/BRNS/3206) Govt. of India, and carried-out at Department of Medical Physics, Anna University, Chennai. The author M.Suresh Kumar would like to acknowledge BRNS for the fellowship and Department of Medical Physics for the facility and Vels Institute of Science, Technology \& Advanced Studies for the support.

\section{REFERENCES}

1. I. Postiglione, A. Chiaviello and G. Palumbo, Cancers, 3(2), 2597(2011), DOI: 10.3390/cancers3022597.

2. G. Manda, M.T. Nechifor and T.M. Neagu, Curr. Chem. Biol., 3(1), 342(2009), DOI: $10.2174 / 2212796810903010022$.

3. G.S. Mohammed Omar, Ph. D. Thesis, University College London, United Kingdom (2010).

4. P.M.L.R. Juliana, A.S.M. Mary, F.G. Sara, B.A. Fernando, R.S. Jackson, A.P.N. Nadia, C.J.Z. Iriana and K.A.R. Lidiyani, Photochemistry and Photobiology. B, Biology, 106, 40(2012), DOI: 10.1016/j.jphotobiol.2011.10.001

5. E. Shacter, Drug Metabolism Review, 32(3-4), 307(2000), DOI: 10.1081/DMR-100102336

6. C.R. Maria and J.C. Robert, Coordination Chemistry Reviews, 233-234, 351(2002), DOI: 10.1016/S0010-8545(02)00034-6.

7. A. Milzani, R. Rossi, P. Di Simplicio, D. Giustarini, R. Colombo, I. Dallendonne, Protein Science, 9(9), 1774(2000), DOI: 10.1110/ps.9.9.1774.

8. Fathi Awad, C. Ramprasath, S. Sivabalan, N. Mathivanan, P. Aruna and S. Ganesan, African Journal of Microbiology Research, 7(37), 4617(2013), DOI: 10.5897/2013.5885.

9. M. Suresh Kumar, B. Anish, R. Murugesan and K. Jeyasubramanian, Nano Biomed. Eng., 4(3), 132(2012), DOI: 10.5101/nbe.v4i3.p132-138. 
RASĀYAN J. Chem.

Vol. 12 | No. 3 |1027 - 1031| July - September | 2019

10. J.J. Correia and H.W. Detrich, Biophysical Tools for Biologists, Academic Press, USA, p. 704, 89 (2008).

11. J. Moan and K. Berg, Photochemistry and Photobiology, 53(4), 549(1991), DOI: 10.1111/j.17511097.1991.tb03669.x.

12. C. Sheng, B.W. Pogue, E. Wang, J.E. Hutchins and P.J. Hoopes, Photochemistry and Photobiology, 79(6), 520(2004), DOI: 10.1562/MU-03-33.1.

13. J.S. Dysart, G. Singh and M.S. Patterson, Photochemistry and Photobiology, 81(1), 196(2005), DOI: 10.1562/2004-07-23-RA-244.

14. M. Suresh Kumar, M. Yuvaraj, P. Aruna, D. Koteeswaran and S. Ganesan, International Journal of Polymeric Materials and Polymeric Biomaterials, 64(10), 519(2015), DOI: 10.1080/00914037.2014.977899.

[RJC-5149/2019] 